\title{
POLÍTICA SELECTIVA DE EPISIOTOMÍA Y RIESGO DE DESGARRO PERINEAL EN UN HOSPITAL UNIVERSITARIO
}

\author{
Selective episiotomy policy and risk of perineal tearing in a teaching hospital \\ Jorge Andrés Rubio, M.D.*
}

Recibido: febrero 8/2005 - Revisado: marzo 28/2005 - Aceptado: mayo 2/2005

\section{RESUMEN}

Objetivo: evaluar el efecto de una intervención educativa tendiente a modificar la política de realización de episiotomía medio lateral y su efecto sobre el riesgo de desgarro perineal durante la atención del parto vaginal espontáneo en un hospital universitario.

Metodología: estudio de cohorte con controles no concurrentes. Lugar: Instituto Materno Infantil, Hospital Universitario de referencia para la atención materno-perinatal. Se incluyeron 3.674 partos vaginales en presentación cefálica entre 20 y 43 semanas de gestación y se excluyeron los partos instrumentados. Desenlaces: frecuencia de episiotomía y de desgarros perineales durante los años 1999-2000 y después de la intervención educativa, durante los años 2002-2003.

Resultados: la política selectiva disminuyó significativamente la tasa global de episiotomía del 47,3\% (IC95\% 41,8-45,7) al 12,2 \% (IC95\% 10,2-14,6) e incrementó el Riesgo Relativo (RR) de desgarro a 1,5 (IC95\% 1,34-1,67) comparado con el basal. El análisis estratificado mostró un leve aumento del riesgo de desgarro para las nulíparas, de 3,83 (IC95\% 2,91-5,03) a 3,90 (IC95\% 2,38$6,44)$ y una disminución significativa de éste, de 4,06 (IC95\% 3,68-6,15) a 0,58 (IC95\% 0,34-0,98) para las multíparas. La política selectiva disminuyó significativamente un 16,4\% (IC95\% 12,7-20,0) la necesidad de sutura perineal posparto.

* Ginecólogo, Profesor Asociado Departamento de Obstetricia y Ginecología Universidad Nacional de Colombia. Dirección: Instituto Materno Infantil Carrera 10 \# 1 - 66 Sur. Bogotá, Colombia. Teléfono: 2333234 Fax: 2333234 Correo electrónico: jarubior@unal.edu.co
Conclusión: la aplicación de la política selectiva de episiotomía fue exitosa: disminuyó la frecuencia de episiotomía sin modificar significativamente el riesgo de desgarro perineal de nulíparas, disminuyó el riesgo de desgarros perineales en las multíparas durante el parto vaginal espontáneo y disminuyó la cantidad de suturas perineales posparto.

Palabras clave: parto, episiotomía, educación, efectos adversos, desgarros.

\section{SUMMARY}

Objective: evaluating the effects of changing routine episiotomy performed in a teaching hospital setting to a selective medium lateral episiotomy policy and its effect on the risk of perineal tearing during spontaneous vaginal delivery.

Methods: cohort study with non-concurrent controls. Setting: Instituto Materno Infantil, a high risk, perinatal reference hospital. Intervention: employing an evidence based selective episiotomy policy. Inclusion criteria: women between 20-43 week gestation attending for spontaneous vaginal cephalic presentation delivery. Exclusion criteria: forceps delivery. Outcomes measured: frequency of episiotomy and perineal tears during 1999-2000 and 2002-2003 following evidence based educational intervention in April 2001.

Results: the selective episiotomy policy significantly decreased the overall rate of episiotomy from $47.3 \%(41.8-45.7,95 \% \mathrm{CI})$ to $12.2 \%(10.2-14.6$, $95 \% \mathrm{CI})$, significantly increased the rate of perineal laceration from $25.1 \%(23.4-26.9,95 \% \mathrm{CI})$ to $37.6 \%(34.5-40.9,95 \% \mathrm{CI})$, especially in nulliparas 
and did not significantly modify overall relative risk (RR) of perineal tearing $(3.58(2.96-4.33,95 \% \mathrm{CI})$ to $2.14(1.44-3.18,95 \% \mathrm{CI}))$. Nullipara perineal tear RR had a non-significant increase from 3.83 $(2.91-5.03,95 \% \mathrm{CI})$ to $3.90(2.38-6.44,95 \% \mathrm{CI})$ whilst it significantly decreased for multiparas (4.06 (3.68-6.15, 95\%CI) to $0.58(0.34-0.98,95 \% \mathrm{CI}))$. Selective episiotomy policy decreased the need for post partum perineal suture by $16.4 \%$ ( $\mathrm{p}<0.05)$. Conclusions: changing the episiotomy policy was successful as selective use decreased the frequency of episiotomy without significantly modifying the risk of perineal tearing in nulliparas and decreased the risk of perineal laceration in multiparas during spontaneous vaginal delivery. The need for post partum perineal sutures also decreased after a selective episiotomy policy had become established.

Key words: delivery, obstetrics, episiotomy, education, adverse effects, lacerations.

\section{INTRODUCCIÓN}

La episiotomía es el procedimiento quirúrgico más realizado en el mundo; se efectúa hasta en el 60\% de todos los partos vaginales y hasta en el 90\% de partos de pacientes primíparas. ${ }^{1,2}$ En nuestro medio, su práctica es generalizada, pues se lleva a cabo en el 87 al 90\% de los partos de las mujeres nulíparas y en el 57 y al $70 \%$ del total de los partos atendidos en instituciones hospitalarias. ${ }^{1,2}$

La episiotomía se ha utilizado en forma rutinaria alegando ventajas durante el parto como la disminución del trauma sobre el piso pélvico, la prevención de laceraciones severas del periné, la prevención de lesiones hipóxico isquémicas en el feto y por favorecer una restauración anatómica y fisiológica del periné. ${ }^{3}$

Sin embargo, los resultados de revisiones sistemáticas y de estudios aleatorizados que han evaluado los beneficios y riesgos de la episiotomía, han cuestionado su uso rutinario, ${ }^{4-6}$ y es así como en la actualidad esta práctica no cuenta con el soporte de la evidencia. Las conclusiones obtenidas, basadas en ensayos clínicos aleatorizados demuestran que la práctica rutinaria de la episiotomía, principalmente la mediana, no previno los desgarros vaginales, ${ }^{5-8}$ ni evitó la aparición de lesiones severas o daño del esfínter anal y por el contrario, sí favoreció la aparición de desgarros perineales leves y de lesiones que comprometen el esfínter y la mucosa rectal. ${ }^{5,9,10}$ Tampoco se encontró que la episiotomía disminuya el trauma sobre el piso pélvico, por lo que no contribuye a la prevención del cistocele ni del rectocele; tampoco ayuda a disminuir la relajación del piso pélvico. Por el contrario, múltiples estudios identifican este procedimiento como un factor de riesgo para desarrollar distopias genitales e incontinencia urinaria de esfuerzo; $;^{7,10}$ también puede retardar el reinicio de la actividad sexual. ${ }^{10-15}$

En cuanto a la duración del período expulsivo del parto, la episiotomía al parecer tampoco lo acorta en forma significativa ni modifica los resultados perinatales. ${ }^{16,17}$ Por el contrario, los estudios antes mencionados informaron un mayor riesgo de complicaciones tales como la extensión hasta el recto y aumento significativo del riesgo de laceraciones complicadas, infección, hemorragia posparto, hematomas, aparición de fístulas, celulitis y fascítis necrotizante, endometriosis perineal, aumento del dolor posparto y en ocasiones resultado anatómico insatisfactorio y dispareunia. ${ }^{5-15}$

Teniendo en cuenta que quienes laboran en el área de partos del Instituto Materno Infantil (IMI) son en su mayoría personas en entrenamiento, el presente estudio se realizó para evaluar el efecto de una intervención educativa, consistente en la difusión de la mejor evidencia disponible, sobre la frecuencia de aplicación de este procedimiento quirúrgico utilizado en la atención del parto y la presencia de desgarros perineales.

\section{MATERIALES Y MÉTODOS}

Diseño: se realizó un estudio de cohorte con controles no concurrentes en el que se comparó la frecuencia de la episiotomía y de los desgarros perineales en un período basal y posterior a la 
implementación de una intervención educativa, que consistió en la difusión de la evidencia disponible respecto a la efectividad del procedimiento al personal encargado de la atención de los partos vaginales, (especialistas, residentes, internos y estudiantes de medicina) en el Instituto Materno Infantil (IMI) de Bogotá, hospital universitario y centro de remisión a nivel nacional. Se respetaron los preceptos éticos emanados por la resolución 8430 de 1993 del Ministerio de Salud y se contó con la aprobación del comité de ética de la institución.

Población: se incluyeron las pacientes que presentaron parto vaginal espontáneo en presentación de vértice, con embarazos entre 21 y 43 semanas de gestación. Se excluyeron del análisis de riesgo de desgarro perineal las pacientes con parto intervenido con espátulas y fórceps por considerarlas con indicación absoluta de realización de episiotomía. Se tomó como período basal de medición previo a la intervención, la frecuencia de uso de episiotomía y de desgarros perineales desde el 1 de enero de 1999 hasta el 31 de diciembre de 2000. Luego se realizó una medición de las mismas variables entre el 1 de enero de 2002 hasta el 31 de diciembre de 2003 como período de comparación post intervención. Intervención: en abril de 2001 se implementó una intervención educativa basada en la difusión de la mejor evidencia disponible relacionada con la práctica de la episiotomía durante la atención del parto. La intervención consistió en la divulgación mediante conferencias, videos, talleres ilustrativos y seguimiento en la sala de partos, así como educación continuada sistemática sobre la atención de parto, de la evidencia disponible a la fecha que favorece la aplicación individualizada y selectiva de la episiotomía medio-lateral en las pacientes independientemente de su paridad.

La decisión de realizar la episiotomía dependió del juicio clínico de quien atendió el parto, basado en la evaluación de la distensibilidad de los tejidos y la longitud del núcleo dorsal del periné para la identificación de las pacientes con inminencia de desgarro vaginal y evitar así el desgarro perineal severo. Se consideró también la urgencia con la que se requería la expulsión fetal, bien por compromiso del bienestar materno o del producto.

Duración de la intervención: 8 meses.

Definición de términos:

Edad gestacional: duración de la gestación a partir de la fecha de la última menstruación confiable o por ecografía del primer o segundo trimestre.

Período basal o de episiotomía rutinaria: corresponde a los años 1999 y 2000, previo a la intervención educativa, en el que se practicaba la episiotomía de manera sistemática para las primigestantes y liberal para las multíparas.

Período de comparación o periodo de episiotomía selectiva: corresponde a los años 2002 y 2003, tras la implementación de la política selectiva de episiotomía para todas las gestantes.

Episiotomía: incisión de la región pudenda con el objeto de ampliar el introito vaginal durante el nacimiento. Esta incisión requiere reparación quirúrgica.

Desgarro perineal: presencia de laceración o disrupción de la mucosa vaginal o de los tejidos de la región pudenda como consecuencia del parto, que requirió reparación quirúrgica.

Necesidad de sutura perineal: reparación quirúrgica del periné, bien por presencia de episiotomía o por desgarro perineal. Involucró el uso de anestesia local infiltrativa y sutura absorbible calibre 2-0. Perímetro cefálico: medición de la circunferencia cefálica fetal en la primera hora posparto, en milímetros.

Peso fetal: medición del peso fetal en la primera hora posparto, en gramos.

Talla fetal: medición de la longitud del feto, desde la coronilla hasta el talón, en la primera hora posparto, en centímetros.

Desenlaces de impacto de la intervención: frecuencia de la realización de la episiotomía y la incidencia de los desgarros perineales.

Los datos se tomaron a partir de los registros de la base de datos del Sistema Informático Perinatal, desarrollado por el Centro Latinoamericano de 
Atención Perinatal (CLAP). Se analizó la información mediante el software Epi Info 2002 (v 3,2) y se compararon las dos cohortes de pacientes, con prueba $t$ student para variables continuas e intervalos de confianza para comparar proporciones. Se estimó el Riesgo Relativo (RR) de presentar desgarro perineal de cualquier tipo durante la atención del parto vaginal espontáneo en ambos períodos, y considerando como exposición la atención del parto sin realizar episiotomía rutinaria, con el objeto de evaluar si el cambio en la política de realización del procedimiento (intervención educativa) modificó la calidad de la atención del parto. Se realizó un análisis estratificado por el antecedente de paridad de las gestantes, el peso, la talla y el perímetro cefálico del recién nacido. Los datos se presentan en tablas y gráficos para su análisis respectivo.

\section{RESULTADOS}

Durante los años 1999 y 2000 se atendieron 2.693 partos y durante el período comprendido entre el 1 de enero de 2002 y el 31 de diciembre de 2003, fueron atendidos 981 partos. Se excluyeron 299 partos instrumentados. No se encontraron diferencias significativas desde el punto de vista clínico en las características demográficas de las madres atendidas, sólo se encontró un incremento estadísticamente significativo de las pacientes casadas durante la segunda fase del estudio (tabla 1).

En la tabla 2 se observan las características de los recién nacidos durante ambos períodos. Ya que el promedio de la edad gestacional de los recién nacidos en el segundo período fue significativamente menor, se observó una reducción significativa en el peso, la talla y el perímetro cefálico de los nacidos durante la fase post intervención. No hubo diferencias en la mortalidad fetal ni en el sexo de los recién nacidos.

La tasa de uso de la episiotomía disminuyó significativamente durante el período en el que se llevó a cabo el estudio (gráfica 1). En la segunda fase del estudio se encontró una reducción global de 31,5\% (IC95\% 28,6\%-34,5\%) en el uso de la episiotomía y una reducción del 59,3\% (IC95\% 54,3\%-64,3\%) de su empleo durante la atención de las pacientes nulíparas. La disminución del uso de episiotomía fue significativa para la atención de todas las pacientes con menos de 3 partos previos (tabla 3). Simultáneamente, hubo un incremento en el porcentaje de partos vaginales con presencia de desgarro perineal (gráfica 1). Al comparar los dos períodos de estudio, este incremento fue del 12,5\% (IC95\% 8,9\%-16,2\%), lo cual implicó un riesgo relativo global de desgarro perineal durante el parto vaginal de 1,5 (IC95\% 1,34-1,67) comparado con el período de episiotomía rutinaria. El análisis estratificado por antecedente de paridad reveló que dicho incremento fue significativo a expensas del incremento en la proporción de pacientes nulíparas que presentaron desgarros perineales luego de la intervención (tabla 3).

$\mathrm{Al}$ evaluar el efecto de la realización o no de episiotomía durante la atención del parto sobre el riesgo de desgarro perineal se encontró que éste no se modificó significativamente luego de la intervención, aunque sí disminuyó ligeramente de 3,58 (IC95\% 2,96-4,33) a 2,14 (IC95\% 1,45-3,18) en la segunda fase del estudio.

$\mathrm{Al}$ ajustar por paridad se encontró que durante la primera fase del estudio (período de episiotomía rutinaria), cuando se realizó la atención del parto de nulíparas sin episiotomía, éstas tenían un riesgo relativo de desgarro perineal de 3,83 (IC95\% 2,91-5,03). Luego de la intervención, éste aumentó levemente a 3,90 (IC95\% 2,38-6,41) sin ser significativo. El análisis estratificado para las nulíparas por el peso fetal y el perímetro cefálico de sus recién nacidos, mostró que evitar la episiotomía en estas mujeres fue un factor de riesgo clínica y estadísticamente significativo para la presentación de desgarro perineal en todas las fases del estudio. La talla fetal no constituyó un factor de riesgo para la presentación de desgarros perineales (tabla 4).

En las pacientes multíparas se encontró que durante la primera fase del estudio, la atención del parto sin episiotomía constituía un factor de riesgo para desgarro perineal (RR 4,06; IC95\% 2,68-6,15). En el segundo período el riesgo de 
Tabla 1. Características demográficas de las pacientes atendidas en el Instituto Materno Infantil por parto vaginal. Período 1999-2003.

\begin{tabular}{|c|c|c|c|c|}
\hline \multirow[t]{3}{*}{ Año } & \multicolumn{2}{|c|}{ 1999-2000 Política rutinaria } & \multicolumn{2}{|c|}{ 2002-2003 Política selectiva } \\
\hline & \multicolumn{2}{|c|}{$n=2.693$} & \multicolumn{2}{|c|}{$\mathrm{n}=981$} \\
\hline & Promedio & IC95\% & Promedio & IC95\% \\
\hline Edad materna (años) & 24,1 & $23,8-24,3$ & 23,9 & $23,4-24,3$ \\
\hline Nivel educativo & n (\%) & IC95\% & n (\%) & IC95\% \\
\hline Analfabetismo & $20(0,7)$ & $0,5-1,2$ & $15(1,5)$ & $0,9-2,6$ \\
\hline Primaria & $696(25,8)$ & $24,2-27,6$ & $236(24,1)$ & $21,4-26,9$ \\
\hline Secundaria & $1591(59,1)$ & $57,2-60,9$ & $626(63,8)$ & $60,7-66,8$ \\
\hline Universitario & $91(3,4)$ & $2,7-4,2$ & $49(5,0)$ & $3,8-6,6$ \\
\hline Sin dato & $295(11,0)$ & $9,8-12,2$ & $55(5,6)$ & $4,3-7,3$ \\
\hline \multicolumn{5}{|l|}{ Estado civil } \\
\hline Soltera & $338(12,6)$ & $11,3-13,9$ & $100(10,2)$ & $8,4-12,3$ \\
\hline Unión libre & $1523(56,6)$ & $54,7-58,4$ & $573(58,4)$ & $55,2-61,5$ \\
\hline Casada & $494(18,3)$ & $16,9-19,9$ & $231(23,5)$ & $20,9-26,4$ \\
\hline Otro & $26(1,0)$ & $0,6-1,4$ & $12(1,2)$ & $0,7-2,2$ \\
\hline Sin dato & $312(11,6)$ & $10,4-12,9$ & $65(6,6)$ & $5,2-8,4$ \\
\hline \multicolumn{5}{|l|}{ Paridad previa } \\
\hline P0 & $1.169(43,4)$ & $41,5-45,3$ & $437(44,5)$ & $41,4-47,7$ \\
\hline P1 & $633(23,5)$ & $21,9-25,2$ & $222(22,6)$ & $20,1-25,4$ \\
\hline $\mathrm{P} 2$ & $466(17,3)$ & $15,9-18,8$ & $161(16,4)$ & $14,2-18,9$ \\
\hline P3 & $224(8,3)$ & $7,3-8,4$ & $82(8,4)$ & $6,7-10,3$ \\
\hline P4 & $116(4,3)$ & $3,6-5,2$ & $39(4,0)$ & $2,9-5,4$ \\
\hline P5 & $50(1,9)$ & $1,4-2,5$ & $30(3,1)$ & $2,1-4,4$ \\
\hline$P>=6$ & $35(1,3)$ & $0,0-1,4$ & $10(1,0)$ & $0,0-1,3$ \\
\hline \multicolumn{5}{|l|}{ Vía del parto } \\
\hline Instrumentado & $235(8,7)$ & $7,7-9,9$ & $64(6,5)$ & $5,1-8,3$ \\
\hline Eutócico & $2.458(91,3)$ & $90,1-92,3$ & $917(93,5)$ & $91,7-94,9$ \\
\hline
\end{tabular}

IC95\%= Intervalo de Confianza 95\%. * $=t$ student .

desgarro perineal de las multigestantes disminuyó significativamente. Luego de la intervención educativa, atender el parto evitando la episiotomía fue un factor protector para los desgarros perineales (RR 0,58; IC95\% 0,34-0,98) en estas pacientes. Estos resultados fueron consistentes al realizar el análisis estratificado por peso y perímetro cefálico de los recién nacidos (tabla 4).

Se encontró una disminución estadísticamente significativa del 16,4\% (IC95\% 12,7-20,0) en la proporción de pacientes que requirieron sutura perineal posparto luego de la intervención educativa. Durante el período basal, el 90,8\% de las pacientes nulíparas requerían sutura perineal luego del parto; el porcentaje disminuyó a $66,5 \%$ durante la segunda fase. De igual forma, la necesidad de sutura perineal posparto en multíparas disminuyó un 13,2\% al aplicar la política selectiva. En ambos casos, la reducción de la necesidad de sutura tuvo significancia estadística (tabla 5). 
Tabla 2. Características de los recién nacidos de las pacientes atendidas en el Instituto Materno Infantil por parto vaginal. Período 1999-2003.

\begin{tabular}{|c|c|c|c|c|}
\hline \multirow[t]{3}{*}{ Año } & \multicolumn{2}{|c|}{ 1999-2000 Política rutinaria } & \multicolumn{2}{|c|}{ 2002-2003 Política selectiva } \\
\hline & \multicolumn{2}{|c|}{$\mathrm{n}=2.693$} & \multicolumn{2}{|c|}{$\mathrm{n}=981$} \\
\hline & Promedio & IC95\% & Promedio & IC95\% \\
\hline Semanas de gestación & 37,5 & $37,4-37,6$ & 36,6 & $36,3-36,8$ \\
\hline Peso RN (gr) & 2.907 & $2.885-2.928$ & 2679 & $2.638-2.720$ \\
\hline Talla RN (mm) & 487 & $486,2-488,7$ & 482 & $479,7-484,7$ \\
\hline Perímetro cefálico (mm) & 336 & $335,1-336,6$ & 332 & $329,7-333,4$ \\
\hline Mortalidad fetal & n (\%) & IC95\% & n (\%) & IC95\% \\
\hline Anteparto & $28(1,1)$ & $0,6-1,4$ & $17(1,7)$ & $1,0-2,8$ \\
\hline Intraparto & $3(0,1)$ & $0,0-0,4$ & $0(0)$ & $0,0-0,5$ \\
\hline Vivo & $2.662(98,8)$ & $98,3-99,2$ & $964(98,8)$ & $97,1-98,9$ \\
\hline \multicolumn{5}{|l|}{ Sexo recién nacido } \\
\hline Masculino & $1.408(52,3)$ & $50,4-54,2$ & $501(51,1)$ & $47,9-54,2$ \\
\hline Femenino & $1.283(47,6)$ & $45,7-49,5$ & $477(48,6)$ & $45,5-51,8$ \\
\hline Por definir & $2(0,1)$ & $0,0-0,3$ & $3(0,3)$ & $0,1-1,0$ \\
\hline
\end{tabular}

IC95\%= Intervalo de Confianza 95\%. * $=t$ student

Tabla 3. Tasa de uso de episiotomía y presencia de desgarros perineales luego de parto vaginal espontáneo, según antecedente de paridad, en el Instituto Materno Infantil. 1999-2003.

\begin{tabular}{|c|c|c|c|c|}
\hline Año & \multicolumn{2}{|c|}{ 1999-2000 Política rutinaria } & \multicolumn{2}{|c|}{ 2002-2003 Política selectiva } \\
\hline & \multicolumn{2}{|c|}{$\mathrm{n}=2.693$} & \multicolumn{2}{|c|}{$\mathrm{n}=981$} \\
\hline & \multicolumn{2}{|c|}{ Uso de episiotomía } & \multicolumn{2}{|c|}{ Uso de episiotomía } \\
\hline Paridad & n $(\%)$ & IC95\% & n (\%) & IC95\% \\
\hline P0 & $839(84,4)$ & $82,0-86,6$ & $99(25,1)$ & $21,0-29,8$ \\
\hline P1 & $186(30,9)$ & $27,3-34,8$ & $11(5,2)$ & $2,6-9,1$ \\
\hline $\mathrm{P} 2$ & $37(8,3)$ & $6,0-11,3$ & $2(1,3)$ & $0,2-4,6$ \\
\hline $\mathrm{P}>=3$ & $13(3,1)$ & $1,7-5,4$ & $0(0)$ & $0,0-2,3$ \\
\hline Total & $1.075(43,7)$ & $41,8-45,7$ & $112(12,2)$ & $10,2-14,6$ \\
\hline Paridad & \multicolumn{2}{|c|}{$\begin{array}{l}\text { Parto con presencia } \\
\text { de desgarro }\end{array}$} & \multicolumn{2}{|c|}{$\begin{array}{c}\text { Parto con presencia } \\
\text { de desgarro }\end{array}$} \\
\hline P0 & $152(15,3)$ & $13,1-17,7$ & $177(44,9)$ & $40,0-50,0$ \\
\hline P1 & $199(33,1)$ & $29,4-37,1$ & $84(39,8)$ & $33,2-46,8$ \\
\hline $\mathrm{P} 2$ & $167(37,4)$ & $32,9-42,0$ & $52(33,2)$ & $26,4-41,8$ \\
\hline $\mathrm{P}>=3$ & $99(23,8)$ & $19,8-28,2$ & $32(20,3)$ & $14,3-27,4$ \\
\hline Total & $617(25,1)$ & $23,4-26,9$ & $345(37,6)$ & $34,5-40,9$ \\
\hline
\end{tabular}

$\mathrm{P}=$ Paridad. IC95\%= Intervalo de Confianza 95\%. 
Tabla 4. Riesgo de desgarro perineal por atención de parto vaginal espontáneo. Instituto Materno Infantil. Período 1999-2003.

\section{NULÍPARAS}

\section{Global}

\begin{tabular}{|l|c|c|c|c|c|c|}
\hline & \multicolumn{3}{|c|}{ Presencia de desgarro } & \multicolumn{3}{|c|}{ Presencia de desgarro } \\
\hline Sin episiotomía & Sí & No & Total & Sí & No & Total \\
\hline Con episiotomía & 63 & 92 & 155 & 163 & 132 & 295 \\
\hline & 89 & 750 & 839 & 14 & 85 & 99 \\
\hline
\end{tabular}

\section{Peso fetal $>3.000$ gramos}

\begin{tabular}{|l|c|c|c|c|c|c|}
\hline & \multicolumn{3}{|c|}{ Presencia de desgarro } & \multicolumn{3}{c|}{ Presencia de desgarro } \\
\hline Sin episiotomía & Sí & No & Total & Sí & No & Total \\
\hline Con episiotomía & 26 & 28 & 54 & 39 & 23 & 62 \\
\hline & 45 & 331 & 376 & 9 & 30 & 39 \\
\hline
\end{tabular}

\section{$\mathrm{PC}>34,0 \mathrm{~mm}$}

\begin{tabular}{|c|c|c|c|c|c|c|}
\hline & \multicolumn{3}{|c|}{ Presencia de desgarro } & \multicolumn{3}{|c|}{ Presencia de desgarro } \\
\hline & Sí & No & Total & Sí & No & Total \\
\hline Sin episiotomía & 26 & 26 & 52 & 60 & 23 & 83 \\
\hline \multirow[t]{2}{*}{ Con episiotomía } & 50 & 299 & 349 & 7 & 19 & 26 \\
\hline & \multicolumn{3}{|c|}{ RR 3,49 (IC95\% 2,40-5,07) } & \multicolumn{3}{|c|}{ RR 2,68 (IC95\% 1,41-5,13) } \\
\hline
\end{tabular}

\section{Global}

\begin{tabular}{|l|c|c|c|c|c|c|}
\hline & \multicolumn{3}{|c|}{ Presencia de desgarro } & \multicolumn{3}{|c|}{ Presencia de desgarro } \\
\hline & Sí & No & Total & Sí & No & Total \\
\hline Sin episiotomía & 444 & 784 & 1228 & 161 & 349 & 510 \\
\hline Con episiotomía & 21 & 215 & 236 & 7 & 6 & 13 \\
\hline
\end{tabular}

\section{Peso fetal $>3.000$ gramos}

\begin{tabular}{|l|c|c|c|c|c|c|}
\hline & \multicolumn{3}{|c|}{ Presencia de desgarro } & \multicolumn{3}{|c|}{ Presencia de desgarro } \\
\hline Sin episiotomía & Sí & No & Total & Sí & No & Total \\
\hline Con episiotomía & 271 & 382 & 653 & 85 & 113 & 198 \\
\hline & 15 & 104 & 119 & 5 & 1 & 6 \\
\hline
\end{tabular}

PC $>34,0 \mathrm{~mm}$

\begin{tabular}{|l|c|c|c|c|c|c|}
\hline & \multicolumn{3}{|c|}{ Presencia de desgarro } & \multicolumn{3}{|c|}{ Presencia de desgarro } \\
\hline Sin episiotomía & Sí & No & Total & Sí & No & Total \\
\hline Con episiotomía & 267 & 387 & 654 & 74 & 116 & 190 \\
\hline & 13 & 113 & 126 & 5 & 0 & 5 \\
\hline
\end{tabular}

\begin{tabular}{|l|c|c|c|c|c|c|}
\hline \multicolumn{4}{|c|}{ RIESGO GLOBAL DE DESGARRO EN PARTO VAGINAL ESPONTÁNEO } \\
\hline & \multicolumn{3}{|c|}{ Presencia de desgarro } & \multicolumn{3}{|c|}{ Presencia de desgarro } \\
\hline & Sí & No & Total & Sí & No & Total \\
\hline Sin episiotomía & 507 & 876 & 1383 & 324 & 481 & 805 \\
\hline Con episiotomía & 110 & 965 & 1075 & 21 & 91 & 112 \\
\hline & RR: 3,58 (IC95\% 2,96-4,33) & RR: 2,14 (IC95\% 1,45-3,18) \\
\hline
\end{tabular}

P.C. $=$ Perímetro cefálico, $\mathrm{mm}=$ milímetros, $\mathrm{RR}=$ Riesgo Relativo. IC 95\%= Intervalo de Confianza 95\% 


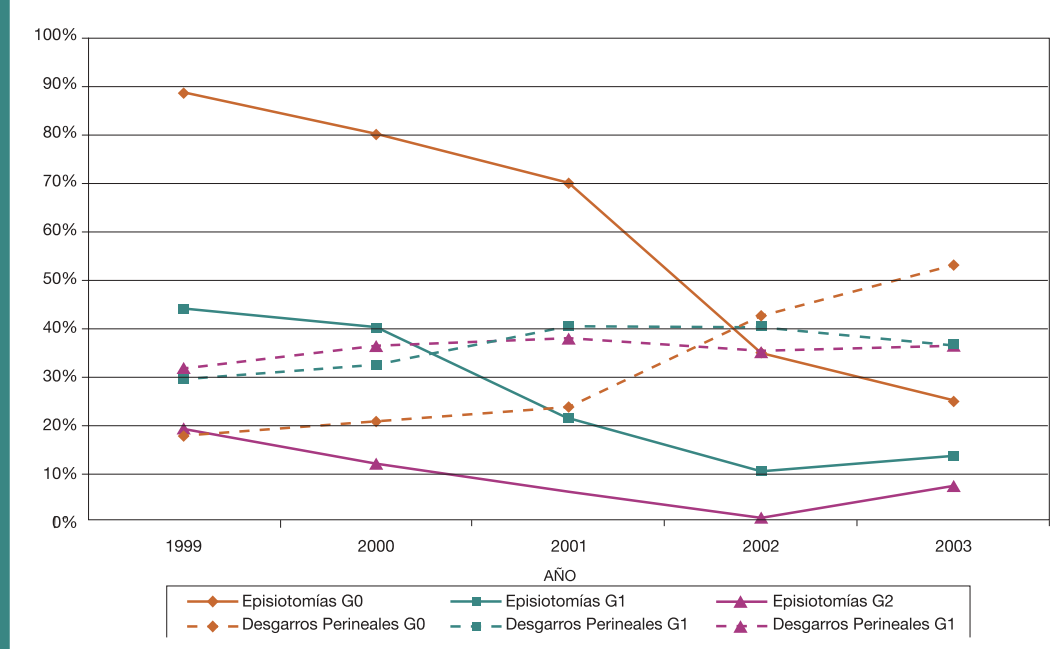

Gráfica 1. Evolución del porcentaje de uso de episiotomía y de desgarros perineales luego de parto vaginal, según antecedente de paridad, en el Instituto Materno Infantil. 1999-2003.

\section{DISCUSIÓN}

Los principales factores determinantes para la realización de episiotomía en el parto vaginal espontáneo son la nuliparidad, el peso y perímetro cefálico fetal. Su uso no se ha asociado con la edad materna ni con la talla fetal. ${ }^{11,19}$

La tasa de uso de episiotomía antes de la aplicación de la política selectiva fue semejante a la reportada en otros países con práctica rutinaria, encontrando tasas mayores del $80 \%{ }^{1}$ y disminuyó significativamente luego de aplicar la política selectiva hasta valores cercanos al 30\%. Este hallazgo es similar al encontrado en el estudio colaborativo realizado en la Argentina. ${ }^{20}$

El incremento de riesgo para desgarro perineal observado es también informado en los estudios de Sleep de 1984 y Belizán, donde al restringir el uso de episiotomía se incrementó el número de desgarros perineales luego de la atención del parto. ${ }^{5,20}$

La menor edad gestacional en el segundo período afectó de manera significativa las variables biométricas de los recién nacidos. Sin embargo, este hecho no disminuyó el riesgo de desgarro perineal de las nulíparas al aplicar la política selectiva de episiotomía, pero pudo contribuir a la reducción del riesgo de desgarro encontrado en las multíparas cuando se restringió el uso de la episiotomía.

Al tratarse de un hospital universitario donde el personal en entrenamiento es responsable de la atención de los partos vaginales espontáneos, existe un riesgo de desgarro perineal mayor durante la atención del parto. Sleep en 1984 encontró un riesgo relativo global de desgarro en partos sin episiotomía de 1,24 (IC95\% 1,07-1,43).

Para las pacientes nulíparas, se encontró un riesgo relativo de desgarro perineal tres (3) veces mayor al reportado en la literatura para este grupo de pacientes y no se modificó a lo largo del estudio. Sleep reportó que el RR de desgarro perineal para primigestantes fue de 1,12 (IC95\% 0,89-1,43) luego de restringir la episiotomía rutinaria. Esta diferencia podría explicarse por el hecho que los partos fueron atendidos por personal en entrenamiento, con menor experiencia y por tanto una menor capacidad de identificar las pacientes con riesgo de desgarro o una inadecuada protección del periné poco distensible propio de las pacientes nulíparas durante la atención del parto. En los estudios referenciados, las pacientes fueron atendidas por parteras o médicos especialistas. La falta de experiencia en la atención del parto ha sido mencionada en otros estudios ${ }^{19,21}$ y junto al peso fetal mayor de 3.500 gramos, el perímetro cefálico mayor de $34 \mathrm{~mm}$ y la edad materna mayor de 31 años, incrementa significativamente el riesgo de desgarros perineales. ${ }^{19,22} \mathrm{El}$ análisis estratificado por peso y perímetro cefálico fetal descartó la presencia de interacción entre tales variables en 
Tabla 5. Pacientes sin necesidad de sutura perineal postparto vaginal espontáneo según paridad, Instituto Materno Infantil. Período 1999-2003.

\begin{tabular}{|l|c|c|c|c|}
\hline & $\mathbf{1 9 9 9 - 2 0 0 0}$ Política rutinaria & 2002-2003 Política selectiva & & \\
\hline & $\mathrm{n}=2.458$ & $\mathrm{n}=917$ & Diferencia & \\
\hline & $\mathrm{n}(\%)$ & $\mathrm{n}(\%)$ & $\%$ & IC 95\% \\
\hline Nulíparas & $92(9,2)$ & $132(33,5)$ & 24,3 & $21,5-27,0$ \\
\hline Multíparas & $784(53,5)$ & $349(66,7)$ & 13,2 & $9,4-16,9$ \\
\hline Total & $901(33,5)$ & $490(49,9)$ & 16,4 & $12,7-20,0$ \\
\hline
\end{tabular}

IC 95\%= Intervalo de Confianza del 95\%.

este estudio. La realización o no de la episiotomía no modificó significativamente el riesgo de desgarro perineal en las nulíparas, pero sí influyó en las características de la lesión que facilitan su reparación y cicatrización. Este procedimiento se ha implicado en el futuro desarrollo de alteraciones del piso pélvico. ${ }^{10,15}$

La reducción significativa del riesgo de desgarro perineal durante la atención del parto sin episiotomía de las pacientes multíparas fue consistente al realizar el análisis estratificado por peso fetal y perímetro cefálico. Esta reducción puede atribuirse a la suma de varios factores: el menor peso fetal encontrado durante la fase post intervención, mejoría en la técnica de la atención del parto para minimizar el trauma sobre el periné materno, (mejor protección del periné, mejor control del expulsivo de la cabeza y de los hombros del feto) y a la mayor distensibilidad del periné de las multíparas. Al comparar con la literatura, el RR de desgarro encontrado para las multíparas es mucho menor que el reportado por Sleep de 1,29 (IC95\% 1,08-1,55). ${ }^{5}$ Estos hallazgos son consecuentes con la literatura que sugiere que evitar la episiotomía disminuye el riesgo de desgarro perineal. ${ }^{5-7,23}$

Los diferentes metanálisis sobre la práctica selectiva de la episiotomía demuestran que esta política disminuye en $44 \%$ la aparición desgarros de la pared vaginal posterior (OR 0,56 IC95\% $0,46-0,65)^{18,20}$ hallazgo que es consistente con los resultados de este estudio para las pacientes multíparas. Simultáneamente, al restringir la práctica de la episiotomía, se aumentan hasta 2,26 veces las lesiones de la pared vaginal anterior. ${ }^{5,20-26}$
En este estudio, se realizó episiotomía mediolateral y no se evaluó la aparición de desgarros vaginales anteriores asociados a la restricción de la episiotomía durante la atención del parto.

La disminución del $16,4 \%$ de la proporción de pacientes que requirieron sutura del periné posparto también es consistente con la literatura. Estudios previos demuestran que la política selectiva de episiotomía permitió una reducción del $28 \%$ en las pacientes con necesidad de realizar sutura perineal. ${ }^{5,20-22}$ Esto representa un ahorro de material quirúrgico, tiempo de atención del parto por el personal médico y para las pacientes menos dolor posparto, ${ }^{5-7}$ un reinicio temprano de labores y comodidad en el momento de atender las necesidades del neonato ${ }^{9}$ y tardíamente, menos dispareunia al reiniciar su actividad sexual. A largo plazo, menor riesgo de incontinencia urinaria de esfuerzo y relajación del piso pélvico. 5,7,9,22-26

Es necesario mejorar la supervisión sobre el personal en entrenamiento y enseñar a reconocer las pacientes con mayor probabilidad de desgarro intraparto para realizar la episiotomía oportunamente, disminuir el traumatismo severo sobre el periné, así como evaluar y mejorar la técnica de protección del periné. ${ }^{27}$ Se deben buscar e implementar alternativas para mejorar la distensión vaginal y perineal (masaje perineal y cambios de posición), que puedan disminuir el riesgo de desgarro durante la atención del parto, principalmente en las pacientes nulíparas o primigestantes.

La implementación de la política selectiva de la episiotomía en un hospital con personal en 
entrenamiento, generó un cambio en la actitud del personal y redujo efectivamente la tasa de episiotomía pero acompañado de un incremento en la frecuencia de desgarros.

\section{AGRADECIMIENTOS}

A los médicos, residentes y estudiantes del Departamento de Obstetricia y Ginecología, por escuchar y modificar sus costumbres, a los doctores Ariel Iván Ruiz Parra y Hernando Gaitán Duarte por sus invaluables observaciones metodológicas y colaboración en el análisis estadístico y a mi esposa, por su inagotable paciencia.

\section{REFERENCIAS}

1. Cravchik S, Munoz DM, Bortman M. [Indications for episiotomy at public maternity clinics in Nequen, Argentina]. Rev Panam Salud Publica 1998;4:26-31.

2. Rubio J. Informe del Departamento de Estadística. Sistema Informático Perinatal. Bogotá: Instituto Materno Infantil; 2001.

3. Gabbe SG, DeLee JB. The prophylactic forceps operation. 1920. Am J Obstet Gynecol 2002;187:254; discussion 255.

4. Banta D, Thacker SB. The risks and benefits of episiotomy: a review. Birth 1982;9:25-30.

5. Sleep J, Grant A, Garcia J, Elbourne D, Spencer J, Chalmers I. West Berkshire perineal management trial. Br Med J (Clin Res Ed) 1984;289:587-90.

6. Harrison RF, Brennan M, North PM, Reed JV, Wickham EA. Is routine episiotomy necessary? Br Med J (Clin Res Ed) 1984;288:1971-5.

7. Klein MC, Gauthier RJ, Jorgensen SH, Robbins JM, Kaczorowski J, Johnson B, Corriveau M, Westreich R, Waghorn K, Gelfand MM, et al. Does episiotomy prevent perineal trauma and pelvic floor relaxation? Online J Curr Clin Trials 1992;Doc No 10.

8. Helwig JT, Thorp JM Jr, Bowes WA Jr. Does midline episiotomy increase the risk of third- and fourth-degree lacerations in operative vaginal deliveries? Obstet Gynecol 1993;82:276-9.

9. Sleep J, Grant A. West Berkshire perineal management trial: three year follow up. Br Med J (Clin Res Ed) 1987;295:749-51.
10. Klein MC, Janssen PA, MacWilliam L, Kaczorowski J, Johnson B. Determinants of vaginal-perineal integrity and pelvic floor functioning in childbirth. Am J Obstet Gynecol 1997;176:403-10.

11. Woolley RJ. Benefits and risks of episiotomy: a review of the English-language literature since 1980. Part I. Obstet Gynecol Surv 1995;50:806-35.

12. Klein MC. Studying episiotomy: when beliefs conflict with science. J Fam Pract 1995;41:483-8.

13. Combs CA, Murphy EL, Laros RK Jr. Factors associated with postpartum hemorrhage with vaginal birth. Obstet Gynecol 1991;77:69-76.

14. Thacker SB, Banta HD. Benefits and risks of episiotomy: an interpretative review of the English language literature, 1860-1980. Obstet Gynecol Surv 1983;38:322-38.

15. Klein MC, Gauthier RJ, Robbins JM, Kaczorowski J, Jorgensen SH, Franco ED, et al. Relationship of episiotomy to perineal trauma and morbidity, sexual dysfunction, and pelvic floor relaxation. Am J Obstet Gynecol 1994;171:591-8.

16. Welch RA, Bottoms SF. Reconsideration of head compression and intraventricular hemorrhage in the vertex very-low-birth-weight fetus. Obstet Gynecol 1986;68:29-34.

17. Barrett JM, Boehm FH, Vaughn WK. The effect of type of delivery on neonatal outcome in singleton infants of birth weight of $1,000 \mathrm{~g}$ or less. JAMA 1983;250:625-9.

18. Gaitán H. Vigilancia Epidemiológica de la Morbilidad y Mortalidad Materna. Noviembre 2002-Octubre 2003. Bogotá: Instituto Materno Infantil; 2004.

19. Robinson JN, Norwitz ER, Cohen AP, McElrath TF, Lieberman ES. Episiotomy, operative vaginal delivery, and significant perinatal trauma in nulliparous women. Am J Obstet Gynecol 1999;181:1180-4.

20.Argentine Episiotomy Trial Collaborative Group. Routine vs selective episiotomy: a randomised controlled trial.. Lancet 1993;342:1517-8.

21. Eason E, Labrecque M, Wells G, Feldman P. Preventing perineal trauma during childbirth: a systematic review. Obstet Gynecol 2000;95:464-71.

22. Carroli G, Belizan J. Episiotomy for vaginal birth [Cochrane Review]. In The Cochrane Library, Issue 2, Oxford, Update Software, 2003.

23. Anthony S, Buitendijk SE, Zondervan KT, van Rijssel EJ, Verkerk PH. Episiotomies and the occurrence 
of severe perineal lacerations. Br J Obstet Gynaecol 1994;101:1064-7.

24. Gass MS, Dunn C, Stys SJ. Effect of episiotomy on the frequency of vaginal outlet lacerations. J Reprod Med 1986;31:240-4.

25. Buchan PC, Nicholls JA. Pain after episiotomy--a comparison of two methods of repair. J R Coll Gen Pract 1980;30:297-300.
26. Angioli R, Gomez-Marin O, Cantuaria G, O'sullivan MJ. Severe perineal lacerations during vaginal delivery: the University of Miami experience. Am J Obstet Gynecol 2000;182:1083-5.

27. McCandlish R, Bowler U, van Asten H, Berridge G, Winter C, Sames L, et al. A randomised controlled trial of care of the perineum during second stage of normal labour. Br J Obstet Gynaecol 1998;105:1262-72. 